\title{
Trivium
}

Revue franco-allemande de sciences humaines et sociales - Deutsch-französische Zeitschrift für Geistesund Sozialwissenschaften

$31 \mid 2020$

La culture politique de la République romaine

\section{Res publica amissa : retour sur la construction d'un paradigme}

Introduction à la nouvelle édition de 1980

\section{Christian Meier}

Traducteur : Anne-Laure Vignaux

\section{OpenEdition \\ Journals}

Édition électronique

URL : https://journals.openedition.org/trivium/7041

DOI : 10.4000/trivium.7041

ISBN : 1963-1820

ISSN : 1963-1820

Éditeur

Les éditions de la Maison des sciences de l'Homme

Référence électronique

Christian Meier, «Res publica amissa: retour sur la construction d'un paradigme », Trivium [En ligne], 31 | 2020, mis en ligne le 30 juin 2020, consulté le 23 août 2021. URL : http://journals.openedition.org/ trivium/7041; DOI : https://doi.org/10.4000/trivium.7041

Ce document a été généré automatiquement le 23 août 2021.

\section{(c) $(1)$}

Les contenus des la revue Trivium sont mis à disposition selon les termes de la Licence Creative Commons Attribution - Pas d'Utilisation Commerciale - Pas de Modification 4.0 International. 


\section{Res publica amissa : retour sur la construction d'un paradigme}

Introduction à la nouvelle édition de 1980

\section{Christian Meier}

Traduction : Anne-Laure Vignaux

\section{NOTE DE L'ÉDITEUR}

\section{Édition originale | Originalausgabe}

Res publica amissa. Ein Studie zu Verfassung und Geschichte der späten römischen Republik, Wiesbaden, Franz Steiner Verlag, 1966 ; nouvelle édition : Francfort-sur-le-Main, Suhrkamp, 1980, p. XIV-LVII.

Nous remercions M. Christian Meier de nous avoir accordé l'autorisation de traduire ce texte pour le présent numéro.

Wir danken Herrn Christian Meier für die freundliche Genehmigung, diesen Artikel in französischer Übersetzung zu publizieren.

Pour la traduction, un certain nombre de coupes a été réalisé dans le texte par JeanMichel David. Les passages supprimés sont indiqués par des crochets [...]; à certains endroits, la rédaction a ajouté une phrase (entre parenthèses et en italiques) pour créer une transition. Les chiffres romains entre crochets (de XIV à LVII) renvoient aux pages de l'édition allemande de 1980 . Seule une petite partie des notes de bas de page a été conservée

A state without the means of some change is without the means of its conservation.

E. Burke, E. : Reflexions on the Revolution in France, Londres, 1967, p. 191. 


\section{La concentration sur le politique}

1 [XV] [...] Mon intention première était de comprendre la façon dont la République romaine avait sombré. Mais lorsque j'ai examiné dans ce but les explications les plus sérieuses qui avaient déjà été avancées, sa faillite m'a toutefois paru moins étonnante que sa très longue existence. Au point que, progressivement, une deuxième question a pris le pas sur la première, celle de savoir comment cette chose commune [Gemeinwesen], cette res publica amissa avait pu fonctionner si longtemps. Je me suis donc concentré sur sa structure.

2 La voie que j'ai empruntée dans ma recherche de la solution était celle de l'identification la plus concrète possible des interdépendances politiques. Je ne pouvais pas me contenter de considérer comme virulents les dysfonctionnements sociaux, les graves conflits politiques - parfois sanglants -, les changements de constitution, les violations et les écarts commis à son égard, l'échec des institutions confrontées à la multiplicité des tâches, la résistance à toute réforme, les divergences - et, semblerait-il, les contradictions - entre ordre traditionnel et nouvelles réalités, la corruption et l'ensemble des autres «facteurs » de naufrage habituellement évoqués. Il me semblait plus important d'enquêter sur les répercussions véritables de ce que nous cherchons globalement à désigner par ces expressions. Peut-être ce qui nous parait inconciliable ou intenable, ce qui apparaissait déjà en partie insupportable aux contemporains n'était-il en définitive pas si insupportable ou si virulent? Peut-être la dureté de l'impact, la cohérence avec laquelle une chose se contredit ou produit des conséquences diffère-t-elle d'un cas à l'autre? Comment expliquer que des facteurs ayant agi sur une longue période n'ont conduit à la chute de la République qu'après plusieurs décennies - et pourquoi pas bien plus tôt ou bien plus tard ?

3 Bien entendu, chercher à cerner les facteurs nommés jusqu'ici à travers leurs effets concrets n'était pas suffisant en soi. Il fallait surtout réévaluer de la façon la plus fine possible le contexte d'actions et de connexions dans lequel la République tardive s'était perpétuée et modifiée.

4 Ce contexte, je l'ai avant tout cherché dans le politique. Quant aux conditions du politique, je pensais les trouver, d'une part, dans certaines caractéristiques principales de la constitution traditionnelle [überkommene Verfassung] (au sens large du terme), d'autre part, dans les constellations synchrones de l'action et des intérêts politiques (ainsi que des opinions qui les guidaient). [...]

5 [XVI] La concentration sur le politique, dans le sens, cependant, d'une conception entièrement neuve de sa problématique, semble avoir fait ses preuves dans cette matière. Cela a probablement été déterminant pour ma décision de persister dans cette approche [...].

6 Car il me semble encore exact aujourd'hui d'affirmer que la crise de la res publica, quelles qu'aient pu être ses causes, s'est essentiellement déroulée dans le domaine politique. Les domaines économique et social ont bien entendu fourni d'importants prérequis, des matières explosives en quantité, des motivations décisives, des conditiones sine qua non. Par contre, le moment où ils ont agi et leur mode d'action ont été, pendant tout le déroulement de la crise, bien moins tributaires d'eux-mêmes que des constellations particulières et de l'évolution de la constellation politique. C'est dans le registre politique que résidaient le changement, le mécanisme, la dynamique (et son 
accélération) proprement dits du processus de crise. Il n'existe aucune certitude concernant le degré de correspondance de la tendance au changement observée dans ce domaine avec une tendance parallèle dans les domaines économiques et sociaux. Ceux-ci n'ont de toute façon quasiment pas développé de dynamique propre. Pour autant que des changements notables s'y soient produits, ils étaient la plupart du temps provoqués par la politique. [...] Et ces changements ne se sont pas simplement exprimés dans les changements affectant le rôle politique de l'ordre équestre et de la plebs urbana. Lorsque la taille de la plebs lui a momentanément procuré une influence plus forte, dans les années 50 , on ignore totalement si celle-ci n'était pas en fait imputable à P. Clodius ou, surtout, à la situation politique du Sénat, entièrement modifiée par César. [XVII] Le poids politique et le pouvoir de l'ordre équestre n'ont jamais été entièrement fonction de leur situation économique. De plus, s'il s'avérait que le rôle des vétérans avait bel et bien pris de l'importance, cela ne découlait pas, pour autant que nous puissions voir, d'un changement intervenu au sein de la plèbe rurale (dans laquelle ils étaient recrutés comme soldats), mais bien des modifications de rapports de pouvoir entre le Sénat et les grands individus, d'une hausse des exigences conditionnée par la politique, bref, en un certain sens, d'un changement de mentalité. À cet égard, le rôle du " grand individu ", du général éminent, s'est révélé comme un facteur structurant. On ne peut pas non plus nier les défis qui ont résulté par exemple de la détresse des paysans avant 133 ou des différentes famines (par exemple suite à la chute des importations due à la piraterie maritime avant 67); dans l'ensemble toutefois, ce n'est pas le changement économique ou social qui a pesé sur la politique, mais bien les changements intervenus dans le domaine politique qui ont conditionné l'usage fait des réalités socio-économiques. [...]

7 C'est donc là que la crise a trouvé son contexte. Elle a tiré sa dynamique de la lutte opposant l'oligarchie et les grands individus. Elle s'est principalement déroulée dans le changement affectant les rapports de force et les oppositions politiques, la distribution du pouvoir [Machtlagerung], la position du Sénat et ses opposants potentiels, le pouvoir agissant dans et derrière les différentes institutions de la constitution, des prétentions et des attentes ainsi que des mentalités en résultant; elle s'est nourrie de la déformation de rapports auparavant fixes, du changement de culture politique, ou encore du relâchement de la fonction du politique, etc. [XVIII] Ce processus doit par conséquent être réévalué sous l'angle politique. [...]

8 À partir de là, il paraissait également évident de se pencher sur la question de la viabilité, étonnamment forte, de la République du point de vue politique. Cela offrait aussi, à mon sens, une image dans l'ensemble pertinente du fonctionnement politique. Cette division thématique m'a donc semblé si naturelle que je ne l'ai pas définie de manière plus précise ; or, le caractériser par le concept de "Verfassungswirklichkeit » ( « réalité constitutionnelle ») était bien entendu insuffisant et, de surcroît, trompeur ${ }^{1}$. J'aurais dû clairement établir que, dans ma question, il ne s'agissait que de la structure politique de la République tardive et de son fonctionnement et non de la structure de l'ensemble. Parallèlement, la mesure dans laquelle cette nouvelle approche visait un changement thématique dans le traitement de la République tardive n'apparaissait pas de manière suffisamment claire.

9 [XIX] Dans l'immédiat, nous devons encore discuter de ce qui était implicitement entendu ici par "structure politique », c'est-à-dire des aspects de celle-ci qui étaient abordés. Je n'étais en tout cas pas motivé, comme le pensait Jochen Bleicken, par un 
intérêt pour l'État romain correspondant d'une certaine façon à celui de Mommsen pour le droit public. La constitution n'était importante à mes yeux que dans la mesure où elle était liée de manière conditionnée et conditionnante aux traits fondamentaux $\mathrm{du}$ fonctionnement de la structure. D'autre part, une bonne partie de ce que l'on pouvait observer comme problèmes et dysfonctionnements de la société contemporaine, comme crise sociale, pouvait rester en dehors du champ d'étude. Quand par exemple de très nombreux paysans perdaient leur terre ou que des centaines de milliers de démunis vivaient dans une misère noire, cela ne nous dit encore rien en soi de la situation politique. [...] Peter Brunt a affirmé à raison à propos du chapitre concernant la plebs urbana que j'avais sous-estimé cette détresse et la mesure du mécontentement ainsi que le potentiel de pouvoir de cette couche de la population. Mais cela ne faisait que rendre la thèse fondamentale de l'ouvrage plus remarquable, à savoir que la plebs urbana, malgré sa grande détresse et son mécontentement à divers sujets, malgré son immense supériorité numérique, avait très peu de poids politique et évoluait dans le cadre de ce qui existait sans contribuer à la formation d'une alternative ${ }^{2}$.

10 S'agissant des différentes forces, la question se pose ici sur trois plans distincts : (1) ce dont elles étaient capables dans des situations particulières (quand il s'agissait de faire aboutir ceci ou cela). Ensuite, ce qu'elles parvenaient à obtenir au-delà de la situation, soit de manière négative (2), si elles participaient, en tant que facteur perturbant, au processus d'affaiblissement général, soit de manière positive (3), si elles contribuaient directement à la construction d'une alternative à la République. Si nous devons, comme il me semble aujourd'hui, réévaluer vers le haut l'action de la plèbe sur les deux premiers plans, il n'en devient que plus remarquable que celle-ci était pratiquement nulle sur le troisième.

11 Il eût sans doute été opportun, par exemple, de consacrer également un chapitre aux esclaves, d'autant qu'ils ont de temps à autre constitué une menace potentielle à Rome et, à travers leurs révoltes en particulier, représenté un souci pour la politique, voire un élément perturbateur quand celles-ci s'avéraient moins faciles à réprimer. [...] [XX] Si je n'ai pas approfondi le sujet ici, c'est parce que ma thèse s'en serait certes trouvée complétée, mais, en fin de compte, très peu modifiée.

12 Le travail trouve dans la structure et la crise politique de la République tardive un thème homogène et cohérent. Il tente ainsi de se concentrer sur l'examen de ce qui n'avait été traité jusqu'ici que de façon occasionnelle, générale, ou en marge de monographies consacrées à l'histoire sociale et constitutionnelle ${ }^{3}$. [...] Encore faut-il, naturellement, préciser ce que nous entendons ici par structure du politique.

\section{La structure politique romaine au regard des catégories et des attentes de l'époque moderne}

13 En étudiant ce que l'on devait, grossièrement, désigner comme la structure politique de la République tardive, je n'ai pas davantage été guidé par une théorie. J'ai enquêté sur les forces impliquées d'une manière ou d'une autre dans la politique, leurs intérêts, la nature et le ressort de leur participation ou non-participation: les voies de l'action politique, les formes de la politique, les regroupements; les points de conflits et les consensus ; les formes de pensée héritées de la constitution et traditionnelles, ainsi que les limites, imposées par la constitution, par les formes traditionnelles de pensée et par 
leur enchevêtrement, de capacités de planification et d'action de ces forces et, par là même, les possibilités de maintien et de reproduction de ce qui existait. [XXI] Enfin, j'ai tenté de décrire les manifestations et les corrélations particulières, à nos yeux paradoxales à bien des égards, dans l'ensemble et de les comprendre en tant que phénomène d'extensification.

Les explications mécaniques des situations invoquant la propension au conservatisme des Romains, les institutions constitutionnelles, la puissance tirée de ses clientèles par la noblesse semblaient - et me semblent encore - insuffisantes pour expliquer cet étonnant attachement à la tradition et, conséquence de celui-ci, la remarquable impuissance de la République face à ses problèmes. [...]

15 Je suis initialement parti de l'hypothèse selon laquelle le maintien et le changement s'expliquaient par les actes des protagonistes politiques. En faisant cela, j'ai, de manière plus ou moins naïve, transposé à la Rome ancienne des attentes modernes: parmi celles-ci, il y avait par exemple l'idée que les grands changements devaient découler de l'émergence d'une alternative à la situation existante, comme dans les révolutions des temps modernes, et qu'en cas de faillite d'une aristocratie, une large couche sociale, en premier lieu celle des chevaliers, devait incarner cette alternative, dans laquelle, éventuellement, l'idée de monarchie prendrait racine. Une autre attente était liée à la précédente, à savoir que lorsque la forme et la destinée d'une communauté étaient en jeu, les citoyens et en particulier les politiques devaient être guidés par les intérêts de toute la communauté - autrement dit par des intérêts politiques - et non par des points de vue "privés "; que l'ensemble menacé, devenu la tâche, devait, sous une forme quelconque, devenir l'objet de la politique et d'oppositions politiques. [...]

[XXII] Tout d'abord, j'aurais dû faire preuve d'une plus grande rigueur terminologique. Car si, de ce point de vue, l'étonnante distance du quotidien romain par rapport au processus de crise qui s'en nourrissait pouvait être expliqué de manière pertinente, il était fort peu approprié de qualifier les mobiles agissant dans cette partie de la politique de "privés", même entre guillemets. Dans le cadre de la société aristocratique, tout établissement de liens familiaux et tout acte conforme à ceux-ci étaient entièrement politiques, même s'ils ne concernaient pas la res publica dans son ensemble.

il aurait fallu éviter une série d'imprécisions découlant de la transposition trop rapide de catégories modernes à la Rome de la fin de la République. La distinction, propre aux temps modernes, entre État et société, par exemple, est dans l'ensemble inappropriée pour cette époque. On peut même se demander si le seul usage de la notion d'État pour les époques prémodernes ne favorise pas l'introduction d'une quantité de fausses associations. En tout cas, la société ne peut, dans ce sens très limité, se définir que par opposition à l'État des temps modernes. [...]

Comme s'il y avait eu une séparation entre État et société durant la République tardive ! D'accord, l'intention transparaît plus ou moins dans le propos: la société conçue comme "somme des individus » devenait le « véritable point de référence de l'action politique »; [XXIII] «La politique intérieure dans son ensemble s'était transformée en une suite d'actions visant à équilibrer les intérêts particuliers ». Mais cela ne démontre pas une réelle compréhension.

19 Pour saisir adéquatement les problématiques dont il est question ici, il est préférable de développer d'autres catégories, plus spécifiques. Puisque cela touche à une thématique 
centrale de la structure de la République romaine, il nous faut nous y arrêter un peu plus longuement.

Pour commencer, il faut souligner que sous l'effet de cette terminologie moderne erronée, deux réalités très différentes ont été confondues dans le texte. Premièrement, nous avons affaire à un trait caractéristique de la constitution romaine qui doit être compris, en quelque sorte, comme le manque de dissociation d'un ordre politique par rapport à l'ensemble de la société (plutôt que comme l'unité de l'État et de la société, ou l'« étatisation» de la société). Il s'agit d'une caractéristique déterminante de la "gewachsene Verfassung ", d'une constitution qui s'est formée à travers l'histoire, étape par étape, une caractéristique qu'il n'est pas facile de bien définir de manière positive. La deuxième réalité est l'orientation particulière de la citoyenneté romaine vers l'intérêt général de la res publica, une forme particulière d'attachement vis-à-vis d'une série de missions et d'obligations. Ces deux données ne sont pas nécessairement liées, mais, dans le cas présent, elles le sont étroitement, génétiquement, et elles s'influencent mutuellement d'une façon spécifique, qui va dans le sens du maintien et $\mathrm{du}$ fonctionnement de la constitution.

Le changement qui mène à la République tardive se caractérise donc (plutôt que par une " sape du caractère étatique ») par un affaiblissement notable de l'orientation vers l'intérêt général au profit de l'orientation vers les intérêts particuliers, par l'avènement de profonds antagonismes, et, en ce qui concerne la constitution, par l'impuissance particulière d'un ordre politique insuffisamment dissocié. Cependant, rien ne change, même alors, à la réalité de ce manque de dissociation. En d'autres termes: l'homogénéité ne subit aucune rupture, mais plutôt une extensification. Rome reste, en dépit de toutes les crises, fidèle à sa constitution telle qu'elle s'était développée au cours du temps [gewachsene Verfassung].

Pour préciser les catégories de "dissociation de l'ordre politique ", de "gewachsene Verfassung " et d'" homogénéité ", une comparaison avec la naissance de la démocratie athénienne s'avère être ce qu'il y a de plus rapide. Dans celle-ci, un ordre politique a été dissocié de l'ensemble de la société. Malgré la persistance de l'inégalité sociale, un tout nouveau rapport entre citoyens, reposant sur l'équilibre, a été instauré dans le domaine politique et fonctionnait effectivement. Une telle atteinte à la correspondance entre constitutions politique et sociale n'est possible qu'au moyen d'institutions qui, au bout du compte, confèrent une force politique à ceux qui sont socialement faibles. De larges couches de citoyens devaient pouvoir accéder au centre de l'ordre politique et ce centre devait avant tout être disponible intellectuellement et pratiquement pour les nondirigeants. La souveraineté sur le bien commun devait être transférée à l'ensemble des citoyens, mieux l'ordre institutionnel tout entier devait être réagencé. [XXIV] La cité [Bürgerschaft] devait ni plus ni moins se réinventer pour qu'à l'avenir ses membres se voient essentiellement comme des citoyens et tiennent compte de cette donnée; sans quoi, ils ne pourraient pas peser sur les décisions politiques. De cette façon, ils étaient intégrés dans un rapport tout neuf, intense, et construisaient ensemble une nouvelle unité, une unité qualitativement nouvelle. [...]

Dans la République romaine, en revanche, l'ordre politique traditionnel a bien été modifié à plusieurs reprises dans les détails. Mais le cœur de la constitution, la question de savoir qui domine, n'y a jamais été soumise à débat. Jamais on n'y a construit de système cohérent d'institutions secondaires. Jamais la cité [Bürgerschaft] ne s'y est reconstituée de manière fondamentale. On n'est pas allé plus loin, dans cette direction, 
que la fondation de la conjuratio de la plèbe. Un pouvoir politique y a bien entendu été créé à partir de la détresse sociale et de l'impuissance, mais ce pouvoir se contentait de modifications dans l'ordre traditionnel.

Si l'on veut exprimer la même chose de manière positive, il faut d'abord constater qu'à Rome, ce qui est transmis de génération en génération est dans l'ensemble toujours conçu comme "pré-donné ». [...] Cette constitution ne tirait pas sa cohérence d'un système d'organes et de règles, mais de l'ordre politique d'une société façonnée d'une manière déterminée par le religieux, le culturel, le social et l'économique. [XXV] Cela signifie que nous avons affaire à une création extrêmement complexe, dans laquelle ce que nous distinguons de nos jours comme politique, religieux, social ou économique (et qui est chez nous réellement à distinguer, fût-ce dans une mesure décroissante, grâce à la dissociation du politique par rapport aux autres domaines) y est inextricablement mêlé. Les rapports de forces objectifs, les rapports de clientèle, le savoir social de multiples natures ne relevaient pas, à Rome, de la sphère de la constitution (comme conditions de leur possibilité) ni de sa réalité, mais ils se trouvaient en même temps au centre de l'ordre, dont ils étaient des parties immédiates, totalement incontournables ou indissociables. Les institutions et règles les plus diverses en présupposaient l'existence ${ }^{4}$. [...]

Ce type de constitution est désignée plus loin dans l'ouvrage comme "constitution naturelle » [gewachsene Verfassung], mais n'est pas traitée plus en détail. De manière plus abstraite, on pourrait parler de « constitution nomiste ». Si l'on cherche à formuler avec précision la pensée politique spécifique qui va de pair, la formule qui semble la plus adéquate est probablement celle d'« homogénéité du savoir politique ». [XXVI] Il s'agit de l'accord peu explicité, mais plutôt pré-donné, partagé entre le haut et le bas sur ce qu'est l'ordre juste. Il s'enracine dans un enchevêtrement relativement dense d'attentes, d'accomplissements personnels et d'attentes d'attentes ainsi que de définitions et de limitations de l'action qui en résultent. Elle ne permet que peu de distance par rapport à la réalité. Elle est certes conciliable avec les conflits, y compris les affrontements violents, voire sanglants. Mais ceux-ci ne peuvent jamais ébranler que des parties de l'ensemble (celles qui concernent les protagonistes respectifs) et non l'ensemble lui-même, qui reste prédéterminé. La conjuratio de la plèbe est également restée particulière, sa solidarité n'étant pas devenue suffisamment globale et fondamentale pour ne pouvoir se réaliser qu'au sein d'une toute nouvelle constitution.

Ce concept d'homogénéité permet également de mieux saisir les changements du savoir, là où, de la même façon qu'en Grèce, ce savoir était de plus en plus secoué, où l'on constatait dans l'ensemble une discordance entre bon ordre et statu quo, où une distance croissante vis-à-vis de la réalité devenait possible, où donc aussi, les germes d'une hétérogénéité du savoir politique se développaient - jusqu’à ce que l'homogénéité se brise et que, dans le domaine politique, on remplaçait l'ancien ordre par quelque chose d'entièrement neuf. À Rome, en revanche, la déstabilisation a eu lieu sans que, pendant très longtemps, les prémisses d'une hétérogénéité, d'une alternative, se dessinent. C'est là que résidait l'impuissance particulière de la République tardive. On peut bien entendu se demander si le problème du manque d'alternative a réellement pris des proportions bien plus grandes à cause d'elle. En tout cas, il s'y manifestait de manière nettement plus claire (ce qui rend la Rome de l'époque tellement exemplaire). 

de la société dans la République classique. L'autre consistait dans l'orientation relativement forte de la citoyenneté vers les missions et les obligations bénéficiant au bien commun. Les multiples dangers extérieurs incitaient à cela et la façon dont on abordait ces problèmes offrait une telle quantité d'exemples et de modèles et procurait à ceux qui s'y engageaient une telle force de persuasion que cette configuration doit s'être stabilisée dans l'enchevêtrement d'attentes, d'accomplissement de soi et d'attentes d'attentes. C'est ainsi que l'échelle des valeurs politiques s'est objectivée, que les instances capables de les incarner et de les inculquer sont devenues puissantes. [...] Nous ne devons toutefois pas nous méprendre à propos de cette orientation relativement forte vers l'intérêt général. En effet, elle n'a pas été à même de dissuader les Romains de cette époque de promouvoir leurs intérêts personnels et ceux de leurs lignées avec vigueur et imagination. Ils ne s'en sont certainement pas privés. [XXVII] En revanche, la cause de la res publica, qu'elle soit prise dans son ensemble ou (ce qui représente l'autre aspect de la question) au niveau des instances dans lesquelles elle se concentrait et qui avaient par conséquent pour mission de la définir, doit avoir été assez forte pour qu'il y ait eu une remarquable convergence entre intérêts généraux et particuliers. L'engagement en faveur du bien général était manifestement honoré d'une considération, d'une influence et d'un pouvoir tels que les particuliers réalisaient leurs prétentions entièrement personnelles ou familiales dans l'accomplissement politique et militaire. Parallèlement, une pression a dû s'exercer pour les empêcher de pousser trop loin leurs exigences, concrètement, à un niveau tel que la solidarité d'ordre et la position dominante du Sénat s'en seraient trouvées menacées. Des circonstances très diverses interagissaient ici : le pouvoir de certaines règles, l'autorité des principes et leur capacité de rester unis, qui imposaient leur respect, une série de procédures traditionnelles, qui généraient une combinaison de possibilités d'action et d'unité, mais aussi l'ampleur des tâches (et la force de Rome, qui permettait de les accomplir en suivant des lignes de conduite traditionnelles), et, last but not least, l'intérêt de l'ordre : avec bien entendu son corollaire négatif, l'absence de matière à conflit plus importante. Dans la République classique, de nombreux intérêts particuliers n'ont certainement pas non plus manqué de contrecarrer l'orientation vers l'intérêt général ou, en tout cas, de n'en tenir guère compte. [...] Même en se montrant très prudent, on pouvait prendre pour une attitude ou une prestation volontairement éthique propre à un peuple entier ou à son aristocratie ce qui était en réalité en bonne partie l'affaire d'institutions déterminées.

peut se demander si des termes comme "bonne volonté » ou "s'en remettre à » (comme je l'ai fait aux pages 45, 52 et 58 du livre) décrivent correctement l'attitude du peuple romain face au leadership de son aristocratie. [XXVIII] Sans doute existait-il d'une manière générale une évidence, régnait-il une confiance relativement élevée dans cette classe. Pourtant, le terme de volonté exige au moins la possibilité d'une mauvaise volonté, chose que nous ne rencontrons que de manière exceptionnelle, dans des situations particulières; fondamentalement, elle n'existait probablement pas. De même, le fait de s'en remettre à quelqu'un suppose une distanciation qui à Rome, n'a certainement jamais été atteinte de la part du peuple vis-à-vis de l'aristocratie. [...]

31 (D'une manière générale, la recherche historiographique doit s'appuyer sur les motivations des acteurs.) 
32 Le respect des obligations, les sacrifices, le dévouement en faveur de l'intérêt commun, avaient alors plus de sens et sont de notre point de vue plus compréhensibles, sans que nous devions pour cela nier qu'il se soit agi de respect des obligations, de sacrifices, de dévouement en faveur de l'intérêt commun.

33 Il serait intéressant de projeter un modèle de ce processus dans lequel le discernement et la force de persuasion d'individus se combinaient à chaque fois avec les nombreuses formes de réactions ponctuelles aux situations ou de conflits dans un système de règles prédéfini, conservant, modifiant ou affinant ce système selon les cas. [...]

34 [XXIX] Le cadre si inhabituel et si étonnant dans lequel on évoluait à Rome livrerait grâce à cela une partie de son mystère. D'autre part, le fait que ces actes soient, au-delà des générations, surtout le fait de principes et de sénateurs moyens deviendrait explicable. Quand il est question de "sagesse sénatoriale », il ne faut pas imaginer une capacité intellectuelle hors du commun dans ce corps, mais plutôt des institutions et des positions qui facilitaient jusqu'à un certain point le fait d'agir avec sagesse (et souvent aussi une supériorité qui menait au succès même quand on n'avait pas agi de manière particulièrement sage au départ). Il en va de même de la capacité unificatrice et de la « discipline » de l'aristocratie.

35 Tout ce dont nous sommes susceptibles de dire que les « Romains avaient ça dans le sang " peut n'avoir été qu'un donné institutionnel préalable "assimilé » et transmis aux individus par le biais de l'éducation, qui a par conséquent caractérisé une longue série de générations de ce peuple, mais qui est non pas née avec le peuple romain, mais plutôt avec son histoire. [...]

$36[\mathrm{XXX}]$ Nous en arrivons ainsi à un troisième point, auquel je m'étais d'abord arrêté en 1966 par le biais de sa version négative, qui résultait d'attentes modernes : la crise sans alternative. En effet, l'absence de solution de remplacement représente, vu avec notre regard actuel, une caractéristique tout à fait déterminante de la crise romaine. Son constat est nécessaire pour orienter l'observateur moderne, à la fois sur Rome et sur ses attentes propres. Sur ce point comme sur d'autres, de nombreuses observations doivent d'abord être rassemblées, évaluées et interprétées, dès lors que l'absence des présupposés que nous avons l'habitude de considérer comme une évidence a été explicitement enregistrée. Les constats négatifs sont alors indispensables pour contrôler le processus méthodologique appliqué. La réflexion sur cette absence d'alternative à la crise peut en elle-même révéler quelque chose d'essentiel de la politique, de la pensée, de la formation des personnalités.

37 Toutefois, le sujet dont il question ici ne se précise réellement que quand la notion même d'alternative est définie comme une constellation particulière d'intérêts, d'actes et d'opinions à l'intérieur d'une crise. Par ailleurs, j'aurais dû approfondir la question suivante: à quel point pouvait-on, dans la Rome de l'époque, compter sur une alternative quelconque; autrement dit, une intéressante propriété structurelle de la société romaine ne devrait-elle pas être définie comme une limite de capacité ? Enfin, il aurait fallu chercher à définir à travers le constat négatif la réalité positive qui s'est présentée à sa place.

38 Si l'argumentation n'a pu franchir de manière plus décisive le niveau de la simple négation des attentes modernes, cela tient au fait que la réflexion sur l'ensemble particulier de la structure romaine n'a pas été menée avec toute la vigueur nécessaire. En dépit de tous mes efforts pour cerner celle-ci et de la quantité d'aspects que j'ai pu 
mettre en lumière, il reste que je n'ai pas pris conscience de la nécessité de délimiter un cadre théorique pour l'ensemble du phénomène. Une fois encore, on peut néanmoins se demander s'il aurait été si facilement possible, dans un seul et même livre, de développer ce travail tellement plus loin que ce qui était donné au départ. Il est en tout cas probable qu'aucune théorie moderne n'aurait pu m'y aider. [XXXI]

Il n'est pas possible d'approfondir ici la question de ce qu'il faut en fait comprendre pertinemment et à la lumière des débats scientifiques modernes comme «structure politique ». Cela nous mènerait bien trop loin. La contribution au thème offerte ici trouve son unité dans la question du fonctionnement de l'ordre politique à caractère aristocratique dans les circonstances de l'extensification. Dans ce cadre, l'accent est mis sur les intérêts politiques, les possibilités de les imposer et la distribution du pouvoir qui en résulte. Une lacune importante doit être signalée à l'intérieur de ce chapitre : l'histoire structurelle du Sénat et de l'aristocratie sénatoriale de la République tardive comme élément particulier de l'extensification aurait mérité un traitement séparé.

Le fait d'avoir largement laissé de côté des questions particulières liées au fonctionnement de la constitution, au rôle des tribunaux, aux problèmes de violence, à l'éducation politique (manifestement en train de se transformer), à l'identité sociale ou encore aux esclaves et à l'administration de la province me parait moins grave : elles auraient sans aucun doute permis d'enrichir l'image générale par d'intéressants traits, mais certainement pas de faire apparaitre quoi que ce soit comme fondamentalement différent.

41 La question de la structure politique incluait aussi celle des frontières du système politique. Dans cette optique, le fonctionnement de ce dernier doit être envisagé non seulement à travers les forces politiques qui y étaient impliquées, mais aussi à travers les tâches qui lui étaient imparties dans le système global. La question de la crise et de la capacité du politique dans la République tardive est ainsi expressément soulevée. [XXXII]

\section{Approches complémentaires}

\section{a) Théorie des regroupements partisans [Parteiungstheorie]}

La difficulté propre à l'étude d'un type très ancien de regroupements politiques tient au fait qu'il est apparemment extrêmement difficile de reconnaître le problème comme tel. L'histoire des sciences montre en tout cas que, dans ce domaine, on a d'abord longtemps été enclin à transposer simplement dans le passé l'image habituelle des groupements politiques de sa propre époque - ou d'une autre, plus familière - et à faire entrer les particularités observées dans le schéma apparemment préétabli. À mesure que la recherche progressait et que de nouvelles données étaient connues concernant les différentes époques, on y apportait des modifications.

Pour la République romaine tardive, cela signifie que l'on imaginait avoir affaire, plutôt qu'à des conservateurs et à des démocrates, qu'à un parti du Sénat ou du peuple, à des factions de l'aristocratie, que l'on pouvait se représenter comme plus ou moins établies et durables. Mais, au fur et à mesure de ce processus, un autre fait propre aux habitudes modernes était considéré comme évident et ne subissait aucune remise en question, à savoir l'idée que ces regroupements (partis ou factions) étaient indépendants des différents objets de la politique. Autrement dit, on supposait que les regroupements 
étaient valables pour la politique dans son ensemble et que les politiciens se retrouvaient régulièrement sur les mêmes fronts indépendamment des objets de la politique. La question de l'éventuelle dépendance de ces groupements vis-à-vis des objets n'a pas été posée. Une fois au moins, on lui a cependant donné une réponse qui s'écartait de l'image habituelle. Pourtant, bien que cette réponse soit venue d'un chercheur aussi estimé que Matthias Gelzer, on ne l'a semble-t-il généralement pas même entendue. Ma propre tentative d'une reconstruction détaillée des formations de partis à l'époque a, je le crois, fourni une image dans l'ensemble pertinente. Mais elle a aussi souffert d'un manque d'approfondissement théorique (ou, pour le dire autrement: les contours de l'ensemble n'ont pas été suffisamment éclairés). [...] [XXXIII]

44 Les fortes variations qu'il peut y avoir entre les formations de partis dans les sociétés aristocratiques face aux thèmes politiques, souvent très particuliers eux aussi, qui se présentent sont notamment illustrées dans la comparaison, exposée plus loin dans le livre (p. 187 et suiv.) entre la République romaine tardive et l'Angleterre du XVIII ${ }^{e}$ siècle. Dans le second cas, ce type de regroupements politiques est indéniablement lié à la présence du Parlement. Néanmoins, comme le montre l'histoire anglaise depuis le $\mathrm{XVII}^{\mathrm{e}}$ siècle, les types de formations les plus divers ont été possibles dans ce Parlement en fonction du rapport à la Couronne, des thèmes politiques et du droit de vote (et, parallèlement, en fonction de l'organisation politique). Pour citer un troisième exemple : ce type de regroupements peut également varier très fort dans une communauté à régime aristocratique dépourvue de Parlement. Une concentration claire de l'influence politique dans un cercle de familles aristocratiques peut donner à celles-ci la liberté de se regrouper en fonction de leurs intérêts familiaux ou encore en fonction des amitiés et inimitiés, ce qui peut à son tour mener à la formation de factions qui se maintiennent pendant quelque temps et dominent toute la politique. [XXXIV] Des forces hégémoniques étrangères comme il y en a eu par moments dans les villes médiévales italiennes peuvent aussi fonder les regroupements sur des oppositions profondes et durables. D'un autre côté, on peut se demander combien de temps le phénomène persiste et même s'il est réellement la règle. Beaucoup d'arguments vont dans l'autre sens : en général, dans les communautés aristocratiques, des parties assez grandes de la noblesse devaient au minimum collaborer (sans quoi, le danger d'éclatement de l'unité aurait été trop grand) et les formations de factions semblent en tout cas généralement n'avoir concerné que de petits cercles. En outre, une aristocratie au pouvoir peut être déterminée par les intérêts divergents de ses partisans à un point tel que des regroupements fixes, indépendants des objets, sont impossibles. [...]

(L'inadéquation du concept moderne de parti tient à la définition de la politique, à la dispersion des centres de décision et à la répartition des forces.)

XXXVIII [...] Enfin, j'ai constaté dans mon texte de 1966 que les groupements politiques romains se constituaient en fonction (ou: par rapport à) des objets de la politique [Gegenstandsabhängigkeit]. Mais le concept (et la distinction explicite par rapport à l'indépendance par rapport aux objets) manque. Un volet de l'enquête se termine en quelque sorte avec cette observation. Je n'ai pas réalisé, ou du moins pas suffisamment, que la véritable différence avec les systèmes de partis modernes et les représentations modernes des partis se situait là et constituait une clé importante pour la compréhension et l'identification du type de regroupements politiques propre à Rome. 
Le concept de dépendance par rapport à l'objet désigne la particularité d'ouverture des différentes forces, qui se groupent en fonction des situations. Les éléments décisifs dans ce cas sont bien entendu l'objet concerné, mais aussi le centre de décision et - cela va de soi - les liens et les motifs objectifs qui entrent en jeu. Le concept de dépendance vis-à-vis de l'objet traduit la différence principale par rapport au système de partis moderne. L'importance du potentiel décisif d'un tel concept central, capturant le trait essentiel d'une chose, ce qu'il apporte en supplément à la compréhension d'un matériel vaste et présenté sous forme de catégories propres, développées spécialement, apparaît de manière particulièrement claire ici.

Le message essentiel et, à ce qu'il me semble, pertinent, concernant le rôle relativement restreint des familles et des factions à Rome aurait par exemple été bien plus facilement explicable et compréhensible dans son ampleur et ses limites si j'avais utilisé cette clé. Il m'avait semblé que le phénomène réellement remarquable, c'était le changement de regroupements d'un sujet à l'autre. Cela m'a amené à sous-estimer les constantes présentes. Cependant, dès que la problématique du rapport entre formations de partis et objets est clairement définie, on s'aperçoit que la variabilité ne constitue qu'un aspect de ce qui est réellement en jeu ici, à savoir la dépendance par rapport à l'objet de ces regroupements. [XXXIX] Il devient alors possible d'apprécier également l'autre aspect, c'est-à-dire les éléments récurrents ou constants.

L'une de ces constantes était l'opposition récurrente de la majorité sénatoriale aux grands projets de loi, autrement dit la division entre optimates et populares (même si ces derniers, en dehors d'un socle permanent, variaient eux aussi généralement d'une situation à l'autre). Ce regroupement n'est décisif que dans les situations exceptionnelles. Lorsque les enjeux sont de moindre importance, il ne se rapporte qu'à des parties limitées de l'agenda politique. Mais en tout cas, il est présent à chaque fois au niveau de l'agitation politique.

Une série de personnalités qui étaient parvenues, à force d'influence et de persuasion, à représenter le centre de la politique sénatoriale entretenaient une collaboration relativement stable. Il y avait dans ce centre une mission continue de "politique constituante ", exercée par des individus comme Catulus et Caton avec un cercle de sénateurs dominants. Certains étaient liés par des liens de parenté, ce qui devait favoriser leur cohésion. Mais ils formaient avant tout le noyau du cercle dirigeant du Sénat. Le pouvoir qu'ils exerçaient ne correspondait que pour une part au « pouvoir de faction » des grands patrons. Il résultait bien plus de bons soins constants, d'un travail de persuasion, d'un engagement pour l'intérêt commun de la majorité sénatoriale, et enfin, d'un facteur qu'il ne faudrait surtout pas sous-estimer : la prime, accordée au sein du Sénat à celui qui défendait de manière conséquente et responsable la cause de la maison. Ces Messieurs incarnaient justement les prétentions du Sénat à la direction, ils avaient de leur côté le poids de la responsabilité sénatoriale à l'égard de la res publica, mais aussi la force que l'on pouvait tirer de l'expérience des échecs divers dans la mesure où les limites du pouvoir sénatorial n'étaient pas définies à l'avance. On ne pouvait que difficilement s'y soustraire. Même les hommes entourant Catulus et Caton ne collaboraient en fait que dans certaines circonstances. Pour de larges pans de l'activité politique, dans lesquelles ce n'était pas jugé indispensable ou urgent, ils ne le faisaient pas ou uniquement sous certaines conditions. Le caractère particulier de leur collaboration ne peut donc être compris que dans le contexte de la dépendance à l'objet des partis. Moyennant cette restriction fondamentale, je peux approuver Erich Gruen 
lorsqu'il qualifie ce groupe de solidaire et d'extraordinaire. Les Metelli et leurs alliés ont dû se trouver dans une situation analogue à l'époque de Sylla (infra, dans le livre, p. 182 et suiv.).

51 Les familles et les connexions familiales constituaient d'une autre façon des constantes dans la politique quotidienne. Elles collaboraient probablement, du moins dans les situations où leurs intérêts communs étaient en jeu. Ces situations étaient cependant assez rares. [XL] On doit bien entendu supposer que l'individu n'était pas seul non plus le reste du temps, qu'il avait au moins sa famille derrière lui en tant que toile de fond et source d'énergie. Sans doute ses amis et ses parents venaient-ils directement à son secours en d'autres circonstances encore. C'est justement lorsqu'en raison de la particularité des thèmes (politiques), seul un petit nombre était directement engagé à travers des obligations qu'il restait une grande marge pour le soutien d'amis et de parents. J'ai sous-estimé cela dans mon exposé. Il reste probablement que, d'une manière générale, les lignées ou même les factions ne pouvaient pas collaborer au moyen de la politique prise dans son ensemble, ni même dans de grosses parties de celle-ci. La particularité des thèmes et l'énorme diversité des relations dont les sénateurs étaient tributaires au fil du temps excluaient certainement que des liens de parenté pussent fournir davantage qu'un support, fût-il renforcé, parmi d'autres. [...]

[XLI] On peut probablement considérer que, dans ces cas, le Sénat pouvait, grâce à la reconnaissance générale de l'ordre traditionnel, apparaître comme le parti qui représentait le tout aux yeux du public. Mais il y avait aussi une condition à cela, à savoir que les oppositions dont il était question n'apparaissent qu'occasionnellement et débouchent sur des regroupements spécifiques. C'est précisément l'alternance de la règle et de l'exception qui a permis pendant si longtemps le maintien de la vieille structure d'origine aristocratique. La démonstration d'unanimité de la majorité sénatoriale dépendait des questions traitées, c'était là sa force et sa faiblesse. Plus précisément : cela causait sa faiblesse dans la mesure où cela le rendait accessible aux principaux intérêts grâce auxquels il pouvait s'affirmer, mais aussi la force, dont il faisait exceptionnellement preuve. Et justement parce que le Sénat n'était pas dans les mains d'une faction ou d'un groupe de factions, sa majorité pouvait, exceptionnellement, agir de manière aussi unanime qu'un parti. S'ajoutait à cela que dans une telle circonstance, la défense de l'ordre traditionnel, qui constituait l'objectif commun, était en jeu.

53 Inversement, le pouvoir de Pompée, si étendu fût-il, était, dans l'ensemble, relativement restreint dans la mesure où faute de cristallisation d'intérêts petits et grands, il ne pouvait pas être durablement intégré dans un regroupement de forces plus large. Il est vrai que les minorités avaient la possibilité de s'imposer de temps à autre avec l'aide de l'assemblée du peuple. Chose que Pompée a faite à plusieurs reprises. Mais même si beaucoup a pu être réalisé par ce biais pour lui et dans certaines circonstances pour les missions de la res publica, au regard de la constitution, cela ne représentait qu'une perturbation : cela créait des rapports de force que l'on ne pouvait pas intégrer dans l'ordre préexistant, car ils contribuaient à sa désintégration. De la même façon, la plebs urbana et les chevaliers (pour peu qu'ils poursuivaient des intérêts communs) représentaient avant tout un potentiel perturbateur. Et ils étaient limités à des effets occasionnels ${ }^{5}$. [...]

54 [XLII] À cet endroit, on peut s'interroger sur l'efficacité de ce système de regroupement partisan. D'une part, celui-ci était à même de satisfaire tous les intérêts puissants 
présents à Rome. De l'autre, il n'atteignait manifestement pas le pensum de la République, c'est-à-dire l'ensemble des tâches dont l'accomplissement était fortement attendu dans une communauté ou, du moins, dont la non-résolution entraînait des conséquences importantes pour sa structure. S'il est intéressant, dans bien des contextes, d'identifier dans une communauté les dysfonctionnements dont les contemporains avaient conscience, il s'agit ici de ceux qui - consciemment ou non, peu importe - étaient virulents et porteurs de crise. Quant à la mesure dans laquelle on peut gérer ces dysfonctionnements dans une communauté, en particulier à travers l'action politique, c'est sans aucun doute une question centrale, décisive, pour la situation de cette communauté. [...]

Il ne s'agit désormais plus de cette caractéristique des regroupements politiques que nous avons appelée Gegenstandsabhängigkeit, c'est-à-dire la dépendance à l'égard de l'objet en débat. En effet, ce système était durant les siècles précédents en général venu à bout des tâches de la République. L'assaut fructueux de la plèbe contre les positions patriciennes a également abouti dans un tel cadre politique. Par ailleurs, les regroupements partisans indépendants de l'objet peuvent probablement aussi s'avérer incapables de mener à bien les réformes nécessaires.

On ne peut pas non plus réduire la capacité de changement du système politique de la République tardive au seul pouvoir des clientèles, des liens de parenté ou des circonstances locales. [XLIII] Pour commencer, on ignore quelle force celles-ci pouvaient avoir, d'autant plus que les liens d'amitié noués librement, quoique devenant obligatoires à plus long terme, prenaient à l'époque progressivement le pas sur les liens de parenté et de clientèle. Ensuite, ainsi que le montre l'exemple grec, de nouvelles solidarités pouvaient, aussi dans le contexte antique, s'imposer au détriment des liens d'amitié et de clientèle aristocratiques.

Il devait donc y voir un autre facteur qui limitait la capacité au changement des regroupements partisans ou, en d'autres mots, la capacité de la société à se grouper pour réaliser la conversion politique des changements en pouvoir correspondant. Dans le livre, la réponse à cette question a notamment été vue dans le fait que ceux qui étaient satisfaits du système étaient trop forts et les insatisfaits trop faibles pour qu'un tel regroupement ait pu s'opérer. Il faut ajouter qu'il était probablement exclu, d'un point de vue structurel, de former une plus large force politique, susceptible d'affronter les problèmes d'une domination d'échelle mondiale. À l'inverse, l'identité républicaine de la citoyenneté romaine ( $\mathrm{du}$ moins dans certaines de ses parties déterminantes) semble avoir défavorisé la mise en œuvre d'une solution monarchique. Ce n'est que tout à la fin, après de longues guerres civiles, que les rapports de force se sont déplacés à ce point qu'Auguste a pu créer une alternative à la situation existante. La grande thématique suivante - comment la crise était-elle mue si elle n'était pas le fruit d'oppositions politiques - est ainsi déjà ébauchée.

Il suffira d'affirmer ceci, en guise de conclusion : si on n'est pas parvenu à Rome à capturer dans la politique les constellations d'actions qui provoquaient les crises, et que, de ce fait, le domaine de ce qui devait être décidé par la politique s'est progressivement restreint par rapport aux tâches, le politique a cessé d'être au centre de la vie commune. Pas forcément au niveau de l'attention, même si celle-ci a également dû baisser, pas tant non plus au niveau du processus de changement, qui s'est, comme nous l'avons dit, essentiellement opéré dans le politique, mais au niveau des effets du pensum. Celui-ci n'était désormais plus réalisé sous la forme de débats et 
de discussions politiques, autrement dit au centre de la société, mais il se constituait sous la forme d'une accumulation d'effets annexes, c'est-à-dire selon un processus.

\section{b) Une crise sans alternative}

Définissons dans un premier temps l'expression de « crise sans alternative ». Désigner certaines situations comme des crises est un procédé à la mode, banal même. [XLIV] Il nous faut donc d'abord un critère pour parvenir à une description plus exacte. Pour plusieurs raisons, le concept de crise semble n'être approprié que quand on a comme point de départ un système. Les crises doivent alors être comprises comme des processus qui mettent un système en danger. Cette mise en danger doit être mesurée à la virulence des facteurs qui génèrent la crise : il doit $\mathrm{y}$ avoir dans le système des constellations sur la base desquelles on agit de telle sorte que le résultat obtenu remet sérieusement son fonctionnement en question ou aboutit à sa dissolution. Cette formulation un peu maladroite a été choisie en considération du fait que des actions ou omissions individuelles peuvent difficilement entraîner à elles seules une crise systémique. Elles peuvent tout au plus y contribuer. Les contextes dans lesquels le système dans son ensemble est pratiqué par ceux qui y prennent part sont déterminants. Dans ce cadre, il s'agit en particulier aussi de la reproduction de prétentions et d'attentes. La virulence des dysfonctionnements tire en effet en bonne partie sa facticité objective de leur institutionnalisation. De ce fait, un même mal peut être tantôt supportable, tantôt virulent; et cela tient précisément au fait que les constellations de l'action sont définies de façons diverses par les prétentions et les attentes. Comme tout concept ou critère, ceux-ci peuvent être maniés de manière fausse ou douteuse.

La question de l'alternative en cas de crise est avant tout liée à la distribution du pouvoir politique [Lagerung der politischen Macht] ou, plus précisément, aux regroupements opérés dans celui-ci en raison de la crise. Cela présuppose la formation de certains potentiels dans le champ de la politique, ce qui n'est peut-être pas toujours possible. Et cela implique aussi une capacité à légitimer de nouvelles configurations. [...]

61 [XLV] Par le mot «alternative » au sens spécifique où il est utilisé ici, il faut donc comprendre, en fin de compte, une force active dans l'espace de la société, qui se manifeste de telle façon que les convictions concernant la nécessité d'une nouvelle configuration s'institutionnalisent suffisamment pour être réalisables dans une mesure significative. Tout ce qui précède peut dans le meilleur des cas être l'ébauche d'une alternative. Il faut encore voir si cela débouchera sur davantage. Les opinions et les intérêts doivent donc se combiner pour former des centres de gravité d'intérêts - et s'installer dans les préoccupations d'un cercle plus large - de manière à ce que leur suivi s'impose à lui, à ce que les opinions et les intérêts correspondants s'objectivent. Nous devons voir s'établir de nouveaux critères, quelle que soit la façon dont ils prennent progressivement forme entre ceux qui mesurent et ceux qui veulent être mesurés grâce à eux. De nouvelles circonstances doivent se dessiner, des circonstances qui exigent une formulation et qui, dans celle-ci, seront finalement offertes à la réflexion et à l'analyse ainsi qu'à une réception de plus en plus large de ces idées.

Un aspect essentiel de la formation d'une alternative réside dans un processus que l'on peut décrire comme «élargissement de l'horizon d'intérêts ». Il présente trois aspects : 
premièrement, il consiste à sensibiliser, autrement dit à augmenter les attentes vis-àvis de l'ordre. Celui-ci devient ensuite apte à produire une alternative si, deuxièmement, il se produit une abstraction des intérêts immédiats des individus en direction d'objectifs plus universels et, si, troisièmement, il se développe dans ce processus une solidarité suffisante pour que l'insatisfaction et la nouvelle vision se matérialisent sous forme de volonté. C'est à cette unique condition que l'intérêt général, dans un premier temps secondaire, peut, face aux intérêts particuliers, devenir central dans un nouvel ordre. Il s'offre, pour ainsi dire, aux occasions de concrétisation. On assiste alors à la formation, dans le champ politique, d'un noyau à partir duquel, avec le temps, la légitimité du système existant (au moins dans ses traits essentiels) est, d'une part, activement remise en question, tandis que, d'autre part, le tout est mieux représenté. Beaucoup de ceux qui sont insatisfaits et cherchent une issue trouvent ainsi une cause vers laquelle ils peuvent se tourner et pour laquelle ils peuvent vivre. Le processus de formation d'alternative se concentre donc dans le politique, mais ses racines et ses répercussions ont une portée bien plus large.

Inversement, aussi longtemps qu'aucune alternative ne se dégage dans une crise, le rapport du système politique correspondant aux problèmes virulents du système global est perturbé. [XLVI] Une crise sans alternative doit donc être identifiée comme une relation déterminée entre actions déclencheuses de crise, virulentes, et le type de regroupements partisans; ou encore, entre mutabilité (déplaisante, non " progressive ») et aptitude à la controverse. Il s'agit de la capacité d'une société à unir et à mobiliser ses forces de sorte qu'il devient possible de faire de la crise elle-même (et pas seulement de certains de ses symptômes) l'objet de la politique (et pas seulement du discours)

64 Il faut souligner l'importance exceptionnelle de la question de l'alternative. Elle est apte à révéler un élément décisif à propos d'une société, de ses possibilités de traitement d'un problème, qui pourraient à leur tour être déterminantes non seulement pour la politique, mais aussi pour les orientations psychiques, les interprétations, la vie spirituelle, la mentalité, bref, l'identité sociale d'une époque. [...] $[\mathrm{XLVII}]^{6}$

$65 \mathrm{Au}$ contraire, le potentiel heuristique du concept de crise sans alternative se révèle exclusivement à la lumière de la question de la causalité du changement et en fonction du rapport entre politique et processus dans ce contexte.

[XLVIII] Pour pouvoir travailler avec ce concept, on doit au minimum tenir compte de la possibilité que, dans une crise, aucune force visant de manière directe et notable un changement ne se dessine pendant un long moment. Quelle qu'en soit la cause réelle, on doit, en toute circonstance, tenir également compte des limites structurelles de la création de pouvoir et de volonté. [...]

Du reste, la crise de la République romaine n'a rien d'un cas isolé dans l'histoire mondiale. Elle présente il est vrai certaines caractéristiques, qui, pour n'être pas uniques, n'en sont pas moins particulières. Elles tiennent à l'« extensification », ce qui signifie que les épisodes de crise n'ont eu pendant longtemps que peu d'effet. S'y ajoutait le fait que pratiquement aucun danger extérieur ne menaçait et qu'il existait une classe relativement large de nantis, qui renforçait la tendance au maintien de la situation existante. Enfin, un rôle était également joué par l'absence spécifique de réactivité, qui découlait des modes de pensée et de la vision de la réalité induits par la constitution telle qu'elle s'était développée [gewachsene Verfassung], ainsi qu'en général, 
par les limites de la capacité politique antique. Toutes ces circonstances réunies font de la crise de la République tardive un modèle particulier.

On trouve dans le texte de mon livre diverses observations à propos de la dynamique processuelle de cette crise. Elles pourraient être élargies de manière significative. Nous nous contenterons toutefois de quelques indications. Différents circuli vitiosi - mieux : spirales négatives - sont observables. Comme celle qui s'est développée entre corruption, enrichissement, élévation des standards, mécontentement et création de tribunaux, et ensuite encore plus de corruption, due à des "prix " plus élevés, et servant entre autres à soudoyer les juges. Ou encore, celle des échecs du Sénat et l'expérience répétée de faiblesse, de résignation, de démonstration de force, de nouveaux échecs, etc. [XLIX] L'accumulation de violations des règles traditionnelles dans les secteurs les plus divers affaiblissait l'unanimité, la cohésion et le pouvoir des sanctions sociales, sur lesquels se fondait le maintien des institutions traditionnelles. L'influence des conditions sous lesquelles les droits étaient garantis décroissait, les moyens de résistance s'usaient progressivement, tandis que la marge de manœuvre des infractions et leur quantité augmentaient.

Si tous ces processus peuvent être observés ou déduits, il n'est guère facile d'en saisir toutes les conséquences. Comme on le sait, une communauté peut vivre longtemps avec la corruption et des organes politiques faibles?

C'est sur l'arrière-plan de ces affaiblissements et d'autres similaires que le processus de crise a trouvé sa véritable dynamique dans un autre contexte encore, à savoir la lutte de l'oligarchie sénatoriale contre des hommes politiques, qui tentaient de s'imposer avec de grands projets. Dans le détail, tout dans ces conflits est contingent. Beaucoup de choses se seraient sans doute passées tout différemment si des certains hommes n'avaient pas agi d'une certaine manière dans certaines situations, s'ils avaient été absents ou si divers enchaînements d'événements ne s'étaient pas parfois percutés de manière aléatoire. La res publica n'était en aucune façon vouée à sombrer à cause de César et à partir de l'an 49.

71 Une certaine structure peut néanmoins être observée dans ces confrontations, qui a, quoi qu'il en soit, probablement dû déterminer le mécanisme spécifique du processus de crise. D'une part, la quantité de dysfonctionnements et de tâches à accomplir devait selon toute vraisemblance permettre à des hommes politiques de mettre en chantier des projets de plus en plus grands, ce qui voulait le plus souvent dire viser le pouvoir à un niveau plus élevé. D'autre part, beaucoup d'indices suggèrent que la majorité sénatoriale s'y opposait avec insistance. Ce point est expliqué plus loin. On pourrait encore ajouter quelques éléments pour expliquer les impératifs institutionnels de la politique de résistance sénatoriale, les opportunités institutionnelles de ceux qu'ils combattaient (voir supra p. XXXIX). On pourrait encore examiner de plus près le processus de raidissement autour de l'ordre traditionnel, qui devenait pour cette raison précise de plus en plus figé. [L] On pourrait développer un modèle d'explication à même de clarifier les multiples échecs et le manque de fortune de l'aristocratie sénatoriale : il serait question $\mathrm{du}$ processus de contraction de la norme sénatoriale, de l'autoprotection méfiante face aux nouvelles réalités à travers l'invocation des Pères et de leur manière d'agir, cela, dans des situations pour lesquelles elle n'était pas faite et dans lesquelles il était impossible de la pratiquer: des répercussions sociopsychologiques d'une faiblesse qui devaient avoir pour effet de décourager des initiatives individuelles, de réduire les possibilités, d'encourager la médiocrité ou au 
moins une droiture obstinée. L'échec de plus en plus manifeste de ceux qui se soumettaient au "politiquement correct» favorisait l'ascension d'hommes exceptionnels. [...]

72 Faute d'une telle alternative, l'ordre établi a peu à peu été anéanti sans être réfuté. Il a été broyé en de violents règlements d'oppositions. Si celles-ci ne visaient pas l'ordre préexistant sur le plan du contenu, elles l'affectaient par ses répercussions indirectes. Si ces nouvelles forces, impossibles à intégrer, ne construisaient aucune alternative, elles perturbaient considérablement le fonctionnement de l'ancien système. [LI] Le monopole sénatorial sur les décisions importantes a fini par être progressivement amolli, les principaux moyens de résistance constitutionnels se sont usés, les conditions pour un maintien en substance des droits constitutionnels ont disparu, la marge de manœuvre de l'action s'est fortement élargie, et, surtout, le pouvoir s'est petit à petit concentré du côté des grands individus. En défendant constamment la constitution sans qu'elle ait été attaquée, le Sénat a précisément poussé des hommes comme Pompée et César à se construire une position à la fois à ses côtés et contre lui. À part ce dernier développement, à l'origine non souhaité comme tel, du moins par Pompée, tout le processus de crise était fait d'accumulations d'effets secondaires non voulus. Car oui, même en ce qui concerne le transfert du pouvoir vers de grands individus, on peut vraiment se demander, en tout cas jusqu'à Auguste, s'il a eu lieu dans la volonté d'instaurer un nouvel ordre, autrement dit, s'il n'a pas également été dans la chute de la res publica un effet secondaire non intentionnel. Quand le fils adoptif de César, le futur Auguste, a fini par viser de manière univoque un nouvel ordre, il a longtemps eu la sagesse de maintenir cette problématique en dehors de l'agenda politique. Répétonsle, jusqu'à son époque, aucune cause s'opposant à l'état existant des choses ne s'était présentée, rien en tout cas qui ait pu servir de point de ralliement à de nombreux intérêts en faveur d'un élargissement de leur gamme.

73 On pourrait il est vrai se demander si l'instauration de la monarchie, qui a finalement apporté la solution, ne devait pas nécessairement se passer de manière personnelle, c'est-à-dire "non objective ». Sauf que, quand les monarchies sont appelées à devenir des alternatives légitimes, elles doivent au minimum se connecter à des causes et s'objectiver à leur contact. C'est ce qui s'est également passé avec Auguste, qui a finalement défini la problématique du bien commun de manière fidèle en mettant en avant la notion de curare à côté de celle de defendere: alors que le Sénat s'était donné comme mission principale la défense de la vieille res publica, Auguste a montré que cette mission consistait à s'occuper de ses problèmes. Enfin, et ce n'est pas le moins important, le besoin de paix, de calme et d'ordre légal devait être satisfait. Mais, bien entendu, le premier princeps a simultanément su se glisser dans le rôle du défenseur de l'ancien ordre et devancer le Sénat, y compris en conservatisme, sous de nouveaux auspices. [...]

74 Il est en tout cas important de constater qu'à Rome, l'alternative ne s'est dessinée qu'après une très longue crise. Cela tenait certainement en bonne partie au fait qu'il n'y existait aucun besoin fort d'efficacité étatique, mais qu'au contraire, une identité républicaine, qui ne pouvait pas si facilement être abandonnée, s'était concentrée dans le Sénat ainsi que, d'une façon ou d'une autre, dans la classe des nantis. [LII] Quelles qu'aient été les solutions de remplacement proposées par Auguste et le degré de participation aux honneurs et aux droits politiques récemment accordés aux couches plus larges des chevaliers (et des Italiques), son régime, dans le cas d'un corps civique si 
fortement orientée vers le Sénat, représentait, malgré tous les avantages offerts, un renoncement sur un point décisif.

Au-delà de ces compléments à la thèse présentée plus loin à propos de l'exemple romain, il faut souligner avec force que la catégorie de la crise sans alternative doit être comprise de manière assez générale. On peut présumer qu'une crise de ce type est également possible en cas de structures dirigeantes incertaines et contestées ainsi qu'en présence de nombreuses oppositions objectives, voire, éventuellement, formulées de manière principielle, à la situation existante. Seul critère obligatoire dans ce cas : les constellations dans lesquelles les hommes agissent dans le système concerné doivent être telles que des actions auxquelles on peut normalement s'attendre déclenchent une crise virulente sans qu'il soit possible de faire de ces constellations l'objet d'une large volonté de changement, en d'autres mots, de les convertir en fin de compte en action politique. [...]

76 Concernant le processus de crise, un point devrait en tout cas s'être considérablement éclairci : le constat d'une absence d'alternative signifie non seulement pour Rome qu'il n'y a pendant très longtemps pas eu de cercle plus large disponible en tant que porteur d'une réforme (ce que l'on savait déjà depuis longtemps) ou que des indices en faveur d'une monarchie se sont faits longtemps attendre (ce que l'on avait découvert depuis un certain temps déjà dans la recherche), mais aussi que nous avons affaire ici à un type de crise particulier, définissable comme un rapport particulier entre mutabilité et aptitude à la controverse, entre processuel et politique, et présentant des propriétés précises. Nous devons nous garder de chercher à comprendre le changement historique uniquement dans la succession des événements et en partant de causes déterminées. [LIII] Il devrait plutôt être possible de distinguer différents modes de changement et, donc, de cerner le problème du changement de manière plus générale.

\section{c) Du problème de la capacité politique}

77 Par capacité politique, il faut comprendre, de manière tout à fait générale, la capacité d'un système à réagir de manière politiquement convenable à toutes les exigences qui nécessitent un traitement commun. Cela comprend notamment l'adaptation du système politique aux changements à partir du moment où cette tâche ne peut être résolue autrement. C'est ce dernier aspect qui nous intéresse ici.

Il semble donc d'autant plus important de se demander, face à ces processus importants affectant l'ordre, jusqu'où s'étend la capacité du système concerné à transformer en objets de décisions politiques les constellations mêmes dont se nourrissent ces processus de changement. Il devrait alors y avoir suffisamment de pouvoir concentré à un même endroit pour introduire des institutions qui, quelle que soit leur nature, permettent de rendre l'ensemble des actions et processus se déroulant au sein d'une société à nouveau conformes à un ordre quelconque, voulu par cette société ou par une majorité de celle-ci.

79 La problématique évoquée ici est apparentée à celle de la « gouvernabilité » dès lors que celle-ci présuppose elle aussi une large base de pouvoir permettant d'appliquer certaines solutions de manière efficace et énergique. Toutefois, la question de la capacité telle qu'elle nous intéresse ici va plus loin. Il faut souligner que l'aptitude d'un régime à imposer des réformes çà et là ne prouve pas en soi qu'il dispose d'une telle capacité. Ces réformes peuvent en effet peser si lourdement sur la structure 
institutionnelle (au sens large du terme) qu'elle en devient moins mobile ou s'en trouve plus fortement perturbée, au point que sa capacité de renouvellement tend plutôt à diminuer. Tite-Live a résumé cet état de fait en lien avec la République tardive par cette courte formule : nec vitia nostra nec remedia pati possumus. ${ }^{8}$

On peut chercher à décrire le problème de la capacité de manières très diverses. On peut parler d'institutions inadéquates, de manque de discernement, de base trop étroite, de capacité d'intégration insuffisante et de bien d'autres choses. Nous laisserons cela en suspens.

Ici, il est surtout question d'un besoin de pouvoir déterminé qui reste inassouvi et, plus précisément, de pouvoir non seulement à l'intérieur des constellations existantes, mais aussi sur celles-ci. Il s'agit à nouveau d'un problème de regroupement des forces. À Rome, l'intérêt général pour le maintien de la res publica existait certes, mais il ne pouvait être invoqué que dans une situation très grave, en cas de senatus consultum ultimum. [LIV] Beaucoup de ceux qui, au nom de leurs ambitions personnelles, posaient continuellement des actes dont les effets secondaires se cumulaient pour affaiblir la constitution pouvaient être mobilisés pour le Sénat. Mais cela ne l'aidait que temporairement ; et on peut franchement se demander si cela aidait la République. Les choses étaient généralement plus graves après qu'avant.

Le manque réel de capacité résidait dans l'absence d'un pouvoir à même de défendre l'ensemble de l'ordre menacé ou de tendre directement vers un ordre entièrement neuf. Cela a débouché sur l'impuissance de la société face à ce qu'elle déclenchait lorsque ses membres faisaient dans leurs constellations d'actions particulières ce que font toujours les hommes : remplir leurs obligations et défendre leurs intérêts, assurer leur survie, pourvoir à leurs besoins, améliorer leurs conditions de vie, tenter d'exploiter les possibilités qui s'offrent à eux, chercher à se distinguer, administrer, faire de la politique, réformer, combattre leurs adversaires, ambitionner le pouvoir.

Bien que le problème consistant à mieux cerner la capacité d'une société en général ne puisse être résolu qu'au prix de recherches comparatives de grande ampleur, on peut quoi qu'il en soit partir d'une distinction fondamentale : l'alternative se forme soit à travers un élargissement, soit à travers un rétrécissement de la disponibilité du pouvoir politique, ou, pour utiliser des termes modernes : à travers un changement allant soit dans le sens d'une démocratisation, soit dans celui d'une oligarchie ou d'une monarchie (abstraction faite de la problématique de la convergence occasionnelle de ces directions).

Dans le cas de l'alternative plus large, la nouvelle répartition du pouvoir, et par conséquent aussi des intérêts est, comme nous l'avons déjà dit, déterminée par un "potentiel d'universalisation", autrement dit la capacité de promouvoir de nouveaux intérêts communs face aux intérêts particuliers et de les ancrer dans des configurations solides. En fin de compte, il s'agit de renforcer l'intérêt commun secondaire pour l'ordre général au détriment des nombreux intérêts particuliers nettement plus évidents. Cela suppose tout d'abord des visions, des prétentions et des positions à partir desquelles ils puissent se développer; ensuite, des possibilités d'organisation comme points de cristallisation pour de nouveaux regroupements ; enfin, une certaine capacité de transformation de l'identité sociale. Car des idées et prétentions pures à un nouvel ordre souhaitable restent facilement académiques et seules des forces déjà présentes, au moins potentiellement, peuvent être organisées. Cette force potentielle doit toutefois résulter d'une façon ou de l'autre de l'enracinement et de la stabilisation des 
visions et des positions dans la «conscience de soi » commune de larges couches - audelà des appartenances habituelles. Cela signifie, bien que je ne puisse le décrire plus exactement à ce stade, que l'identité sociale est d'une certaine façon affectée. [LV] Sa transformation suppose une restructuration de certaines composantes.

À Rome, la possibilité de comprendre la crise ou les conditions préalables pour concevoir un nouvel ordre était très réduite. La constitution traditionnelle telle qu'elle s'était formée au cours des siècles était caractérisée par une imbrication particulièrement étroite dans la réalité, ce qui n'autorisait pratiquement aucune distance par rapport à la situation existante. Parmi les classes supérieures, il n'y avait apparemment pas de positions à partir desquelles une insatisfaction vis-à-vis de la situation existante aurait pu fondamentalement se développer. Quant à construire un nouvel ordre en se reposant sur les classes inférieures, ce n'était pas concevable. Il n'a pu en être autrement que chez les grands individus, qui, pour des raisons diverses, ont visé une position exceptionnelle et puis finalement la monarchie: dans leur cas, l'intérêt personnel a pu se superposer à l'intérêt qu'il y avait à maîtriser la crise. C'était une bonne condition de base pour de nouvelles visions; mais il est difficile de savoir à quel point ces hommes, une fois au pouvoir, ont réellement compris la crise et s'ils ne se sont pas contentés de faire le nécessaire au cas par cas, autant que possible - souvent d'ailleurs en poursuivant simplement leur propre intérêt qui, dans le meilleur des cas, correspondait à l'intérêt général. Auguste, cependant, doit avoir été conscient de la façon dont il pouvait rendre justice aux différentes parties du corps civique et gérer convenablement l'empire.

86 Les capacités limitées des Romains de la République tardive à sortir des schémas de pensée traditionnels ne sont que l'expression, particulièrement visible, d'un certain enfermement de toute l'Antiquité classique dans la réalité. Car au-delà de toutes les différences qui ont pu exister entre Romains et Grecs, il n'y avait à l'époque aucun horizon d'attente concernant la structure du bien commun au-delà d'une certaine amélioration de la situation politique de certaines couches. On ne connaissait pas le rehaussement abstrait des objectifs au nom d'un tout - objectifs qui, en dépit de toute les partialités possibles, avaient tout de même acquis une certaine évidence dans l'histoire des temps modernes lorsque des alternatives à l'ordre existant se créaient. La capacité de définir des objectifs se heurtait donc à un plafond dès que les classes potentiellement puissantes s'étaient assurées de leur part aux droits politiques. Quel que soit l'élargissement des marges de disponibilité ou l'entendement des principes de la constitution, niveaux auxquels les Grecs, au moins, parvenaient, jamais, dans le domaine politique, on n'a franchi le seuil nécessaire pour que la gamme existante des modèles d'ordre politique puisse être considérée comme seulement provisoire et que des attentes d'un futur meilleur, tout différent, puissent être produites. L'idée, si cruciale pour les innovations, qu'un ordre encore existant puisse être dépassé, qu'il ait fait son temps, n'était pas concevable. La nouveauté, la progression temporelle ne pouvait servir d'argument.

87 [LVI] Bien que de manière différente, l'identité sociale antique avait été, chez les Grecs comme chez les Romains, institutionnalisée dans des communes de citoyens, moyennant des modes simples, relativement immédiats de participation à un ensemble politique. Rome avait certes fortement étendu cette identité, mais sans jamais la dissoudre dans une identité nationale, c'est-à-dire médiatisée, englobant la population de plus grandes régions. Dans l'Antiquité, il se pouvait bien entendu que la 
participation des citoyens à la communauté fût plus ou moins concentrée dans certaines couches ou groupes statutaires. Par contre, la séparation entre l'État et la société avec toutes ses spécialisations, abritée sous la coupole de l'identité nationale, n'a jamais existé. La spécialisation, la diversification de la société dans des secteurs relativement autonomes, les distances internes et, en complément à cela, l'identité nationale me semblent en effet liés. Dans l'Antiquité, en revanche, l'identité sociale était en général déterminée par le fait que les citoyens qui portaient la communauté le faisaient précisément en tant que citoyens, c'est-à-dire en mettant fortement en avant leur qualité commune de citoyens et en négligeant les spécialisations ou en n'en développant que très peu (spécialisations que seul un ensemble relativement abstrait aurait été capable d'intégrer).

Étant donné que dans l'Antiquité, le tout était composé de parties concrètes, fixes, non relativisables, l'ordre politique y était synonyme d'être plutôt que de mission ou de moyen. On n'acquérait pas la distance permettant de comprendre le tout de manière suffisamment englobante pour que l'ordre politique puisse apparaître comme partie, comme extérieur. Le tout était si peu abstrait, si peu éloigné que l'on pouvait difficilement se sentir dépendant vis-à-vis de lui et que, par conséquent, il était impossible de le rencontrer avec des attentes aussi élevées (qui peuvent encore voiler idéologiquement leur particularité). En raison du manque de dissociation d'un domaine économique propre, l'idéal de l'efficacité avait également bien du mal à émerger. D'innombrables tâches, par exemple celle d'une politique économique, sociale et éducative, ne se présentaient simplement pas. Même la garantie de l'ordre public était en bonne partie fournie par les citoyens eux-mêmes.

Enfin, il n'y avait pas non plus de possibilités organisationnelles en tant que point de cristallisation pour la formation d'une alternative plus large. L'absence d'idée de constitution représentative (en dehors des fédérations de cités) n'est apparemment que l'expression du fait qu'au-delà des communes de citoyens, on était incapable d'établir un assemblage politique fait de couches plus larges. Cela doit avoir été le revers de l'identité sociale étroite, relativement concrète. Des alternatives plus larges n'existaient donc que dans les États municipaux [Gemeindestaaten]. De ce fait, le potentiel d'universalisation était totalement dépassé étant donné l'ampleur des problèmes et l'étendue déjà immense de l'espace sous la République tardive.

Le résultat final de ces réflexions ne surprendra, soit dit en passant, aucun lecteur bien informé : la seule solution envisageable était la monarchie. [LVII] Ces réflexions n'apprennent donc rien de neuf aux historiens de l'Antiquité. Elles n'ont du sens que si l'on observe le processus (de la crise sans alternative) de l'extérieur, c'est-à-dire à partir du présent, et que l'on cherche à saisir la particularité de Rome (et à la rendre comparable) à partir de catégories générales.

91 La question de la capacité propre à la monarchie en tant qu'autre alternative imaginable va au-delà de la thématique traitée dans ce livre. Quant à l'identité, la monarchie l'a plutôt déconstruite (démontée) et affaiblie. Les principes se sont certes volontiers et de plus en plus reposés sur des membres de classes et de peuples qui n'appartenaient pas encore à la noblesse sénatoriale (ou n'y avaient pas encore fait leur entrée). Mais autant ceux-ci pouvaient fournir de bons magistrats, fiables, autant ils étaient également fixés sur les anciens idéaux aristocratiques. En gros, il s'agissait, à partir du nouveau centre de pouvoir, de démonter les résistances petit à petit, moyennant de nombreuses concessions, et d'introduire de nouvelles idées et critères de 
valeur dans l'ensemble du corps civique. Le nouvel ordre pouvait ainsi se légitimer lentement, assis sur un pouvoir dont les conditions étaient apparues dans la République tardive, mais ne procurant l'alternative proprement dite que quand ce pouvoir était déjà présent, et encore, sous le manteau d'une res publica restituta.

\section{NOTES}

1. [= note 5] Pour une critique, voir par ex. J. Bleicken (in : Savigny Zeitschrift für Rechtsgeschichte, 85, 1968, p. 452 et suiv. et G. Crifo (in : Jura, 18, 1937, p. 239 et suiv.). Aujourd'hui, j'éviterais le concept dans ce contexte. Néanmoins, il faut tenir compte pour la République tardive de l'existence d'une conscience très claire de la différence entre l'ordre tel qu'il était et celui qu'il devait être. C'est ce que voulait dire la note 11 à la page 4 du livre. Durant la période des débuts, l'ordre correct équivalait en revanche à l'ordre réel en dehors du peu qui était combattu au cas par cas parce que jugé abusif ou dangereux. Il est intéressant de se demander comment cette différence peut être saisie du point de vue sémantique dans la constitution naturelle.

2. [= note 8] De façon analogue, voir maintenant E. S. Gruen: The Last Generation of the Roman Republic, Berkeley / Los Angeles / Londres, 1974. Cela reste très frappant, en particulier au vu du manque très large de police (à ce sujet, voir aussi mon commentaire de l'article de A. W. Lintott : "Violence in Republican Rome», Historische Zeitschrift, 213, 1971, p. 395 et suiv.). Car lorsque P. A. Brunt réduit mécaniquement le pouvoir de la nobilitas dans la ville à la taille de sa clientèle, cela peut difficilement être exact (P. A. Brunt: " The Roman Mob », Past \& Present, 35 (1), 1966, p. 3-27, ici p. 21 et suiv.).

3. [= note 10] On trouve une certaine exception à cette règle chez L. R. Taylor: Party Politics in the Age of Caesar, Berkeley / Los Angeles, 1949. Toutefois, il ne s'agit pas d'une observation de la structure du politique, mais d'une étude, très intéressante au demeurant, des techniques politiques (ascension, campagne électorale, alliances, propagande, utilisation de la religion, etc.). Les questions du mode particulier de formation de partis, le rôle politique des ordres et des classes, de la répartition des intérêts, de la situation constitutionnelle particulière et de la dynamique de la crise n'y sont guère abordées de manière approfondie.

4. [= note 20] Cf. Museum Helveticum, 32, 1975 ; Historische Zeitschrift, 213, 1971, p. 395 et suiv. ; A. Peisl / A. Mohler (éd.) : Der Ernstfall, Berlin, 1979, p. 53 et suiv. Quand Brunt (Journal of Roman Studies, 58, 1968) demande ce qui différencie les sénateurs particulièrement ambitieux du $1^{\text {er }}$ siècle de ceux du IV ${ }^{\mathrm{e}}$ siècle, nous devons faire référence non seulement aux possibilités largement développées, mais aussi considérer le relâchement des contrôles et inhibitions sociaux. À cela correspond le rétrécissement des normes, qui sont alors fréquemment violées.

5. [= note 44] Que l'on se rappelle de ce qui a empêché en 58 l'entrée en vigueur de la « concordia ordinum » du Sénat et des chevaliers en faveur de Cicéron. En soi, elle aurait, en correspondance avec l'objet, dû pouvoir aboutir (sans pour cela, ce que Cicéron reconnaissait, pouvoir être indépendante par rapport à l'objet. Cela n'aurait été possible, que si, comme le voulait Cicéron, la défense de la res publica avait réellement été le point de vue déterminant du groupement). Du reste, d'autres différenciations devraient être faites concernant ces groupements. E. Badian (Aufstieg und Niedergang der römischen Welt, éd. par H. Temporini, 1, 1, p. 669) constate par ex. des idiosyncrasies personnelles contre la reconnaissance des alliances entre politiques là où il 
s'agissait en réalité de la différence entre appartenance à un quelconque cercle amical politique et le rôle de représentant et d'outil d'un tel cercle (voir plus loin dans le livre p. 98).

6. [= note 53] J. Gaudemet a fait observer dans son compte rendu (in : Revue historique de droit, 46, 1968, p. 85) que la République avait tout de même produit de nombreuses nouveautés fructueuses, tournées vers l'avenir, comme les imperium extraordinaires et le poids des légions. C'est incontestable. Néanmoins, il s'est d'abord agi avant tout de perturbations; il a fallu attendre le contexte du principat pour que cela devienne les points de départ intéressants et les articulations d'un nouvel ordre. C'est à cela que l'on arrive dans ce contexte. On peut également se demander si le processus de préparation et la fondation de la monarchie, n'auraient pas dû constituer le complément de ce travail sur la République tardive. Voir également Ch. Starr : American Journal of Philology, 89, 1968, p. 483. [...]

7. [= note 54] Voir à ce propos Erich S. Gruen (The Last Generation of the Roman Republic, Berkeley / Los Angeles / Londres, 1974. p. 160). Je n'irais bien entendu pas jusqu'à nier le caractère de crise de ces symptômes et d'autres, analogues. L'œuvre de Gruen constitue, à mon sens, une contribution majeure au débat sur la République tardive. Mais il ne s'interroge pas réellement sur sa structure. Le fait de constater par exemple que les nobiles continuent à être élus - et même, comme je le trouve, plus qu'auparavant - nous informe sur les chances électorales des nobiles, mais pas encore sur le pouvoir des magistrats ou de la nobilitas. Il en va de même du domaine d'autorité des autres segments. L'identification de ce type de constantes ne permet pas de dire grand-chose sur le caractère de crise des situations et, par conséquent, pas non plus le nier.

8. [Tite-Live, Praefatio, 9].

\section{INDEX}

Mots-clés : République romaine, constitution

Schlüsselwörter : römische Republik, Verfassung

\section{AUTEURS}

\section{CHRISTIAN MEIER}

Christian Meier est professeur émérite d'histoire de l'Antiquité à l'université de Munich. Pour plus d'informations, voir la notice suivante. 